\title{
Mechanobiological modelling of tendons: review and future opportunities
}

M.S. Thompson ${ }^{1}$, M.N. Bajuri1,3, H. Khayyeri², and H. Isaksson ${ }^{2}$

5 1. Institute of Biomedical Engineering, University of Oxford, United Kingdom

2. Department of Biomedical Engineering, Lund University, Sweden

3. Faculty of Bioscience and Medical Engineering, Universiti Teknologi Malaysia, Malaysia

Special issue on Computational Modelling in Medicine, Proceedings of the Institution of Mechanical

10 Engineers part H: Journal of Engineering in Medicine

Corresponding author:

Dr. Mark S. Thompson

Institute of Biomedical Engineering,

Department of Engineering Science,

15 University of Oxford,

Parks Road, OX1 3PJ

Oxford. UK

Telephone: +44 1865737845

Fax: +44 1865737640

20 Email: mark.thompson@eng.ox.ac.uk

Keywords: Tendon; tendon healing; tendon mechanobiology; 


\section{Abstract}

Tendons are adapted to carry large, repeated loads and are clinically important for the maintenance of musculoskeletal health in an increasing, actively aging population as well as in elite athletes. Tendons are known to adapt to mechanical loading. Also their healing and disease processes are highly sensitive to mechanical load. Computational modelling approaches developed to capture this mechanobiological adaptation in tendons and other tissues have successfully addressed many important scientific and clinical issues. The aim of this review is to identify techniques and approaches that could be further developed to address tendon related problems. Biomechanical models are identified that capture the multi-level aspects of tendon mechanics.

10 Continuum whole tendon models, both phenomenological and microstructurally motivated, are important to estimate forces during locomotion activities. Fibril level microstructural models are documented that can use these estimated forces to detail local mechanical parameters relevant to cell mechanotransduction. Cell level models able to predict the response to such parameters are also described. A selection of updatable mechanobiological models is presented. These use

15 mechanical signals, often continuum tissue-level, along with rules for tissue change and have been applied successfully in many tissues to predict in vivo and in vitro outcomes. Signals may include scalars derived from the stress or strain tensors, or in poroelasticity also fluid velocity, while adaptation may be represented by changes to elastic modulus, permeability, fibril density or orientation. So far only simple analytical approaches have been applied to tendon mechanobiology.

20 With the development of sophisticated computational mechanobiological models in parallel with reporting more quantitative data from in vivo or clinical mechanobiological studies, e.g. appropriate imaging, biochemical and histological data, this field offers huge potential for future development towards clinical applications. 


\section{Introduction}

Tendons are mechanically extraordinary connective tissues that transmit load from muscle to bone during locomotion. They consist principally of highly aligned, densely packed collagen fibrils embedded in a hydrated "ground substance" of proteoglycans. In humans, tendon disease and

5 injury are common and costly, affecting both professional athletes (UK Achilles tendinopathy estimated incidence 500 per year $^{1}$ ) and the general population (UK Achilles rupture incidence up to $20 / 100,000$ per year ${ }^{2}$ ), with earnings loss and painful and prolonged rehabilitation. In equine athletes, $40 \%-50 \%$ of all injuries are tendon or ligament related, with over $15 \%$ of racehorses suffering from tendon injuries during a training season, this type of injury is the most common 10 reason for retirement of the horse ${ }^{3}$.

Tendon has a multi-unit hierarchical structure, which is mainly composed of type I collagen (60\% dry weight $\left.{ }^{4}\right)$ with a small amount of types III and V. Triple helical tropocollagen molecules are arranged in quasi-crystalline fibrils which run approximately parallel to the tendon's long axis 5 . Fibrils are embedded in a hydrated matrix composed of proteoglycans, glycosaminoglycan (GAG) 15 and glycoproteins - tendons are approximately $70 \%$ water by volume ${ }^{6}$. Fibrils are bundled together as fibres which themselves together with tendon cells form fascicles of which a relatively small number make up a whole tendon ${ }^{7}$. Tendons can withstand repeatedly applied high forces up to five times the human bodyweight during running ${ }^{8}$. The tissue appears to be adapted to resist fatigue loading such that when stress in life was taken into account in ex vivo tests, different

20 tendons from within the same individual were found to fail at approximately the same number of cycles ${ }^{9}$. Fatigue damage is linked to progressive accumulation of discontinuities ('kinks') affecting bundles of fibrils, and the level of damage is associated with the severity of a pathological response and with the development of disease ${ }^{10}$.

Not only are tendons exquisitely designed to provide excellent energy storage in locomotion 25 and precision motion for dexterity; like many other mechanically loaded tissues, tendons are able to adapt to withstand changing loading conditions. Mechanobiology seeks to define the precise 
relationship between mechanical stimulation and cellular and tissue mechanisms which underlie the observed changes in metabolism, structure and mechanical properties ${ }^{5}$. The experimental evidence for tendon's being mechanoresponsive, has been recently reviewed 11,12 .

Understanding healthy and pathological mechanisms for tendon adaptation to mechanical loading is key to developing novel therapies that harness the positive mechanoresponse of the tissue, independent of whether they are pharmacological, mechanical or tissue engineering-based. Moreover, since tendons contain high levels of collagen type I, tendon can act as a simple model system for other more complex, less well-studied collagen based tissues. Finally, the knowledge can also assist in biomimetic design of new highly fatigue resistant, self-healing composite materials.

\section{Motivation and aim of review}

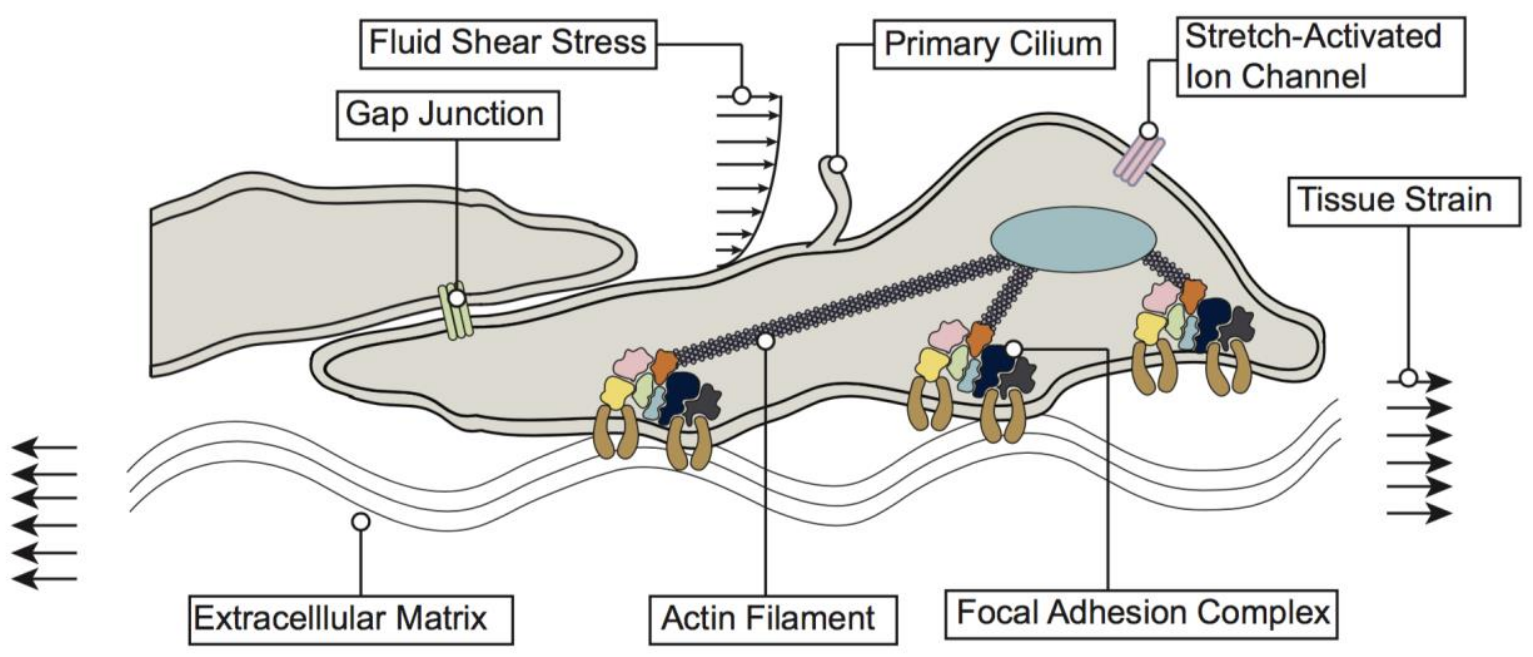

Figure 1. Mechanotransduction mechanisms in tendon. The tendon cell, connected mechanically to its pericellular matrix and to adjacent cells, transduces stretch and fluid shear stress signals through cytoskeletal, membrane and primary cilium mechanisms. With permission ${ }^{13}$.

Precise mechanisms for the tendon adaptive response to mechanical load remain unknown. For tissue adaptation driven by cell activity, understanding the mechanical environment of the living cells and hence the tissue micromechanics are essential pre-requisites. Cells are believed to sense multiple physical signals from the environment through mechanisms sensitive to substrate 
deformation and fluid movement (Fig. 1) and respond anabolically or catabolically. Computational modelling can address these challenges with both 'biomechanical models' that provide a detailed quasi-static picture of the biomechanics of the system at a given time, often focusing on the material model and properties of the tissue, and also with 'adaptive models' that represent the ability of the

5 tissue to remodel over time, predicting the long term mechanobiological adaptation, often trying to identify the cell and tissue response to certain stimuli. The aim of this review is to provide an overview of the latest developments in computational modelling that can be applied to understanding and harnessing tendon biomechanics and mechanobiology and to set out the future directions in this field.

Following recent discussion of nomenclature ${ }^{14}$, this review uses the term fibril to refer to structures consisting of quasi-crystalline collagen which may be up to $500 \mathrm{~nm}$ in diameter. The definition of larger structures may be tissue specific, in tendons fibres are bundles of collagen fibrils and may also contain other protein and cellular components.

\section{Biomechanical models}

\section{Whole tendon}

Whole tendon modelling is essential to define the macro-scale mechanical environment and response of the tissue in biomechanical analysis of locomotion. The mechanical properties of tendons are largely dependent on the way in which the fibril organization changes with loading. The initial toe region ( $0-2 \%$ strain) in a typical tendon stress-strain curve (Fig. 2) represents the 20 stretching-out of the approximately $10 \mu \mathrm{m}$ wavelength fibril crimp-pattern. In the linear region of the stress-strain curve (2\% - 4\% strain) the uncrimped collagen fibrils both extend and also slide past one another ${ }^{15}$. Microscopic damage to fibrils occurs when the tendon is stretched over $4 \%$, whereas strains of $8-10 \%$ result in macroscopic failure ${ }^{16}$. Studies of damage and failure in equine tendons suggest positional tendons fail at lower strains (15-22\%) than energy storing tendons (18$2528 \%)^{7}$. Damage mechanisms also show changes with ageing, with greater damage susceptibility in older energy-storing equine tendons ${ }^{17}$. 


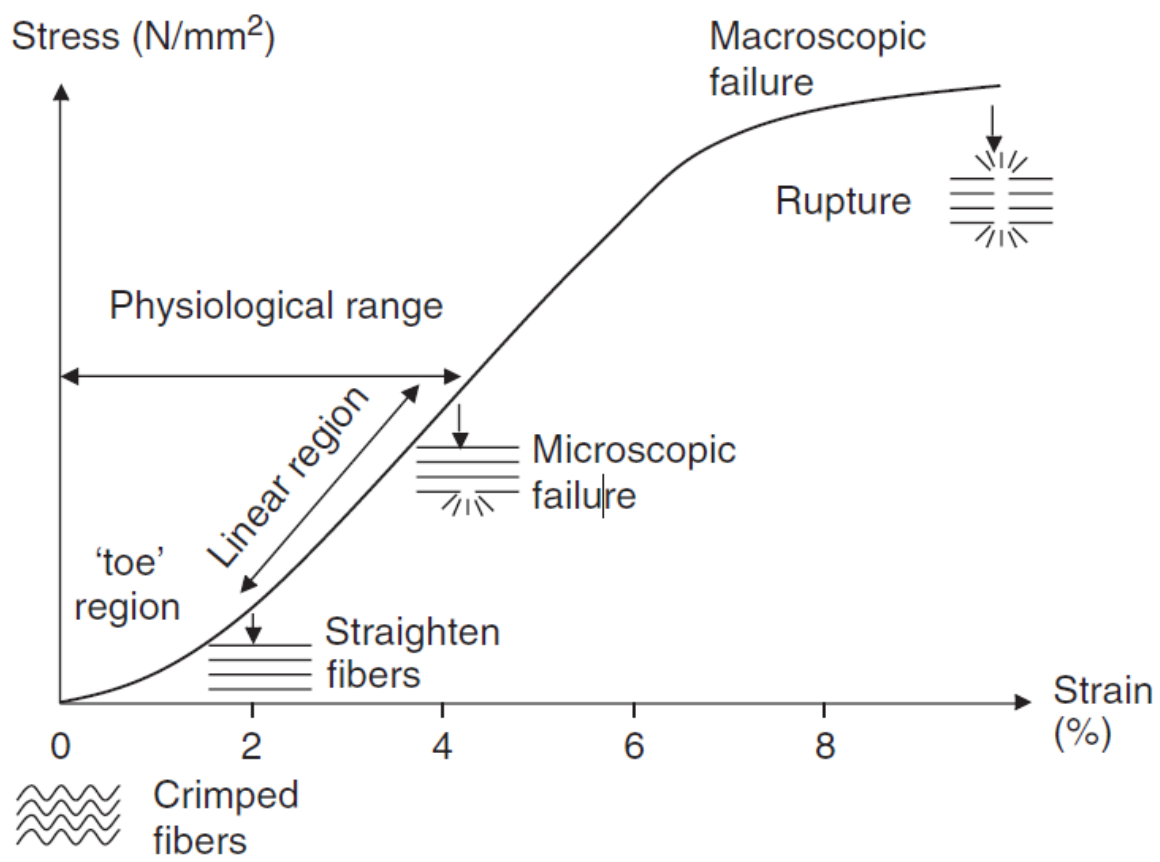

Figure 2. Tendon stress-strain curve with permission ${ }^{5}$

The simplest phenomenological models for the non-linear tendon stress-strain behaviour

5 have used various strain energy functions including exponential ${ }^{18}$, polynomial ${ }^{19}$ and logarithmic 20. Uniform isotropic hyperelastic models have also been used extensively ${ }^{21-23}$. However, as is clear from above the strongly anisotropic fibril orientation and the clear role fibril re-orientation plays in tendon motivate a more sophisticated approach.

Microstructurally motivated continuum approaches have used transversely isotropic

10 hyperelasticity with a single set of fibril orientations to represent tendon and ligament 24 and variants have been used in many finite element implementations for example in supraspinatus 25 and the long head of the biceps tendon ${ }^{26,27}$. Subsequent developments included modelling two distinct families of collagen fibres in cruciate ligaments ${ }^{23}$, and most recently patient-specific Achilles models with realistic geometries ${ }^{28}$. A similar early model for the arterial wall 29,30 has also 15 evolved, incorporating two fibre families each with a symmetric dispersion about their mean orientation angle ${ }^{31}$, which has been successfully applied with a design of experiments approach to represent the mechanical response at discrete timepoints of tendons undergoing tendon healing ${ }^{32}$. These models have also been modified to include a rule-of-mixtures fibre volume fraction ${ }^{33}$. 
Fibril recruitment due to the straightening of crimp has long been considered in models of soft tissue ${ }^{34}$ and continuum models have been proposed with various distribution functions used to represent the recruitment stretch: Weibull ${ }^{35}$, Gamma ${ }^{36}$, or triangular function ${ }^{37,38}$. Most recently a continuum strain energy function was proposed ${ }^{39}$ with a recruitment function derived

5 from considerations of the radial distribution of crimp angle ${ }^{40}$, with good fitting results to patellar tendon data. This strain energy function was further refined to represent the helical arrangement of crimped fibrils although without representation of mechanical interactions between fibrils ${ }^{41}$.

The continuum approach has further been used to identify the importance of mechanical interactions between fibrils and the matrix in which they are embedded to accurately represent 10 biaxial testing of tendons ${ }^{42}$. Further work has suggested that this mechanism for transmission of interfibrillar shear stress is the primary loading mechanism for collagen fibrils in tendon ${ }^{43}$. Another fibre reinforced anisotropic hyperelastic constitutive model has been implemented to study the effect of partial-thickness tear on loading capacities of the supraspinatus tendon ${ }^{44}$. The widespread assumption of incompressibility in soft tissue has been challenged in ligaments ${ }^{45}$, and 15 recent models with slight compressibility have been implemented in finite element analysis ${ }^{46}$.

Viscoelastic models have been developed to capture the time dependent character of tendon behaviour. The most popular, Quasi Linear Viscoelasticity (QLV) uses a hereditary integral formulation often with the exponential strain energy function mentioned earlier 47 48-52. This approach has been developed to implement Mooney Rivlin or Prony series constitutive laws for 20 further efficiency and better fitting at high strains in stress relaxation 5354 . Fully non-linear viscoelasticity has also been applied to capture a wider range of behaviours under physiologically relevant loading 55, 56. Some structurally motivated continuum models including viscoelastic behaviour have been proposed for tendon, where different constitutive equations for different levels of strain have been "patched" together ${ }^{57}$ or using QLV to separately represent the isotropic 25 and anisotropic components of the tissue ${ }^{58}$. X-ray diffraction studies motivated a model using two viscoelastic elements in series to represent molecular friction within the fibril and viscous shear relaxation of the matrix between fibrils respectively ${ }^{59}$. Viscous fibril rearrangement representing 
the contribution of glycosaminoglycan cross links was implemented in fibre-reinforced continuum model ${ }^{60}$ and later extended to consider the damage of these hypothesised cross links ${ }^{61}$. Creep, relaxation and strain stiffening behaviours were captured by a fibre-reinforced Zener model including a recruitment process ${ }^{62}$.

Poroelastic constitutive models, explicitly representing solid and one or more liquid phases, have been successfully used to describe this highly hydrated tissue. Implemented as part of a hyperelastic fibre-reinforced continuum poroviscoelasticity was able to describe both quasi-static and dynamic tests, as well as the direction of the fluid flow in rat Achilles tendons ${ }^{63,64}$.

As mentioned above, whole tendons may be divided into fascicles and the interfascicular 10 matrix is receiving increased attention for its adaptation in tendons with different mechanical function ${ }^{65}$. The mechanics of multiple fascicles can be addressed through wrapping algorithms such as those implemented in musculoskeletal modelling software ${ }^{66}$.

The models presented in the section above assume an affine behaviour of the fibrils in the material and provide a good understanding of the tissue-level behaviour of tendons, especially in

15 the central, approximately linear part of the stress -strain curve. However, only few models could also capture the low strain behaviour governed by processes of fibril recruitment and crimp straightening. Therefore we consider explicit models of fibril mechanics in the next section.

\section{Fibril level models}

Modelling the mechanical behaviour of fibres and fibrils explicitly is essential for

20 understanding tendon mechanobiology since the heterogeneous arrangements of these structures determine the mechanical signals transduced by cells. Initial discrete modelling efforts focused on the crimped pattern of collagen fibrils in tendon tissue, represented as helices ${ }^{67}$, sinusoids ${ }^{68}$, elastica ${ }^{69}$ or with a kinematic chain ${ }^{40,70-72}$. A different approach assigned individual fibrils a bilinear stress strain curve which when integrated over the whole tissue fitted the observed non25 linearity ${ }^{73}$. This theme is continued with recent use of a helical spring model for fibrils to fit whole 
tendon stress - stretch data ${ }^{74}$, acknowledging the limitation of using just one mechanism to account for non-linearity which is known to arise from multiple causes.

Models that include fibril-matrix interaction enable a more sophisticated set of questions to be addressed. Models of the tapered shape ${ }^{75}$ and the slenderness of fibrils ${ }^{76}$ confirmed the role of

5 stress transfer through the matrix for the tissue level properties of tendon. Helically arranged crimped fibrils embedded in a matrix were able to account for both non-linearity and the high Poisson's ratio of tendon ${ }^{77}$. The high Poisson's ratio and fluid exudation under tensile load were addressed by an explicit model of fibrils connected by 'interfibrillar links' embedded in a poroelastic matrix ${ }^{78}$. Together with tensile experiments on enzyme treated tendons a model with 10 straight fibrils with interconnections representing glycosaminoglycans (GAGs) cast doubt on the theory that these components provide interfibrillar shear load transmission ${ }^{79}$. In models of partially-cut tendons a shear lag fibril-matrix load transfer was required to adequately represent the dependence of properties on cut size, and fidelity improved as the number of simulated fibrils increased 80 .

\section{Cell level models}

Tissue microstructure, composition and hence mechanical property adjacent to cells may be different from that of the 'bulk' extracellular matrix. In cartilage, models have shown that this pericellular matrix has an important role in modulating a chondrocyte's environment ${ }^{81}$. An FEA model of tendon including spatially varying fibril recruitment, poroelastic extracellular and highly

20 compliant pericellular matrix estimated the fluid shear stress around ovoid tendon cells ${ }^{82}$.

There is an extensive literature addressing the mechanical modelling of cells. Thus, the reader is referred to an excellent recent review by Rodriguez et al. ${ }^{83}$. For cells remodelling tendon under non-rupture conditions, two important models that could report quantities relevant to the mechanotransduction mechanisms identified in Figure 1 are detailed. An elastic model

25 representing a single cell on a 2D surface including pre-stressed actin and tubulin cytoskeletal elements as well as the nucleus and its actin cortex was able to provide good corroboration with 
AFM indentation experiments on two cell lines before and after cytoskeletal disruption ${ }^{84}$. This model was later modified to include the primary cilium of the cell, a well-known mechanosensory organelle ${ }^{85}$. Another elastic model, aimed at understanding a spherical cell seeded into a collagen gel, represented the cytoplasm, cytoskeleton and nuclear envelope ${ }^{86}$. Cytoskeletal elements were

5 randomly oriented and the effects of uniaxial tension, compression and shear applied to the whole gel were investigated. Counterintuitively, macroscopic uniaxial tension increased cytoskeletal compression, while macroscopic compression increased cytoskeletal tension, and shear appeared not to change the loading pattern.

\section{Adaptive models}

The longest-standing application of adaptive mechanobiological models has been directed towards the understanding of bone tissue during development, repair, and remodelling of bone tissue ${ }^{87-91}$. These iterative approaches use a domain defined in Finite Element Analysis (FEA) to link continuum level mechanical quantities computed from the stress or strain tensors to local tissue change, often represented by change in material properties. This requires the identification 15 of thresholds, defining the ranges of biophysical stimuli for which a particular tissue type can exist 
92. Excellent reviews of this field are available 93,94 .

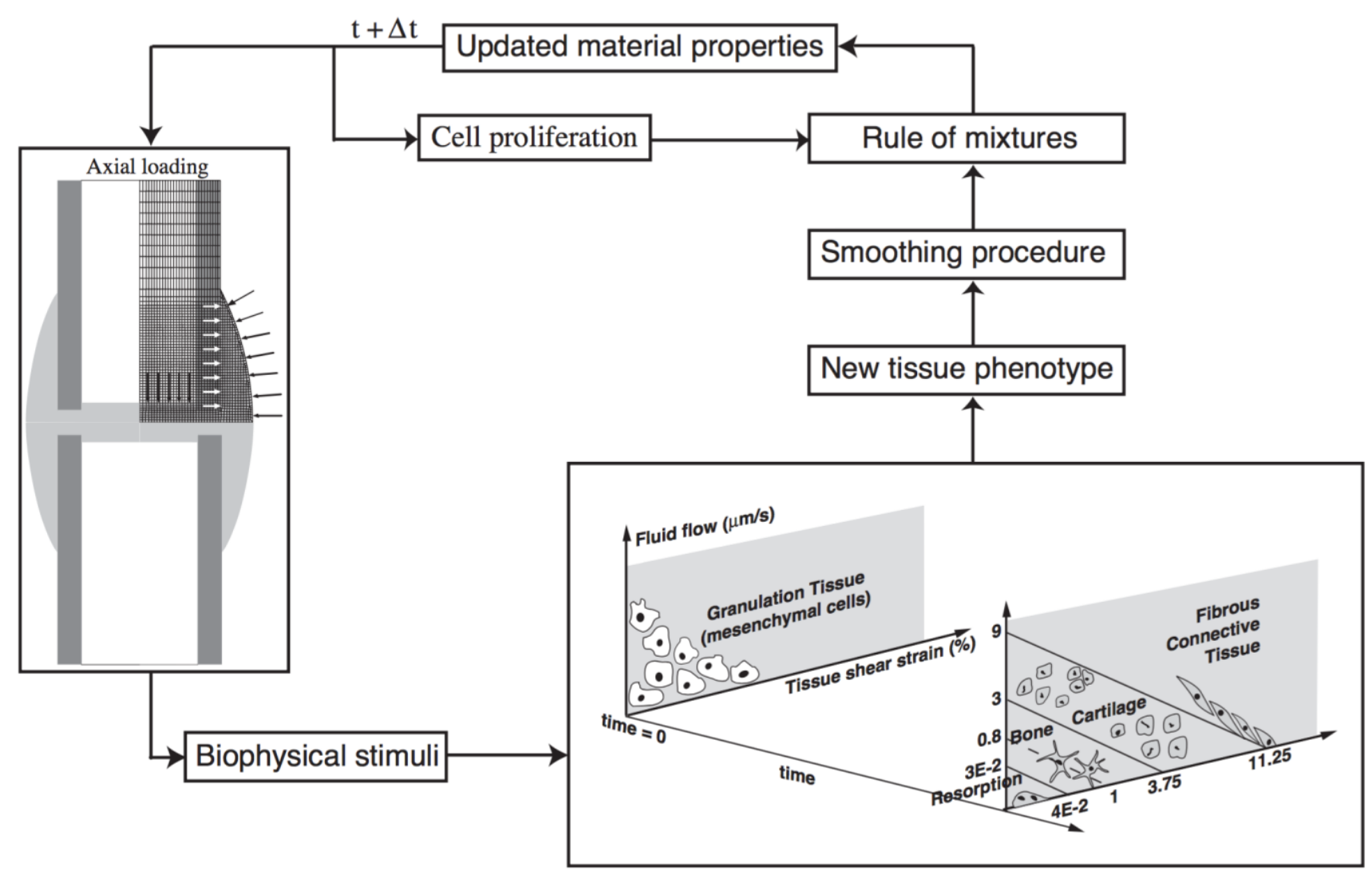

Figure 3. Scheme for bone healing adaptive model - with permission 89

One approach is using a poroelastic finite element model to represent the macroscopic

5 geometry of an animal model for bone healing ${ }^{89}$. Together with thresholds of octahedral shear strain and pore fluid flow, the tissue phenotype of each element and hence its stiffness was updated iteratively (Fig 3). The FEA geometric domain also lends itself to modelling diffusion of growth factors or cells which can then influence the rules for tissue differentiation ${ }^{95,96 . ~ T h e s e ~ m o d e l s ~ a r e ~}$ well accepted to capture bone tissue mechano-response during healing and have been used widely, in vitro for bone regeneration therapy design 97,98 .

A different FEA approach was proposed to study the mechanical conditions that produce dysplasia in the prenatal hip joint, linking the increase in element size in a growth region to octahedral shear stress history ${ }^{99}$. This concept was extended to use an isotropic thermal expansion and remeshing capability to understand the mechanobiological component of joint morphogenesis 15 predicting the role of different movements on formation of hinge and ball and socket joints ${ }^{100}$. The 
same strategy has been used successfully to predict cortical adaptation to externally applied load in mouse tibia ${ }^{101 .}$

An adaptive continuum mechanics approach implemented using FEA has also been used to in plane isotropic model skin expansion for reconstructive surgery ${ }^{102}$. This used a growth tensor 5 proportional to local strain within physiological thresholds and was able to predict patient-specific patterns of skin expansion. Other groups have used the combination of finite element models and a growth tensor formulation to successfully capture rapid embryonic epithelial wound healing 103 . Reaction diffusion equations modelling inflammatory factor and cell migration and collagen deposition in skin wounds using continuum mechanics representations predicted wound shape 10 effects on healing speed ${ }^{104}$.

A different aspect of tissue mechanobiology can be captured by modelling change of fibril orientation using an appropriate constitutive model. Reorientation of collagen fibrils in cartilage due to changing load patterns was represented using FEA and an algorithm rotating the local fibril direction towards an assumed local preferred direction ${ }^{105}$. This approach was further employed in

15 predicting the development of collagen fibril angular distributions in cardiovascular tissues from an assumed uniform distribution under physiological loading ${ }^{33}$. Progressive degeneration of collagen in articular cartilage during development of osteoarthritis was also recently described ${ }^{106}$. In the algorithm, the collagen network stiffness in cartilage was reduced iteratively when a maximum principal stress criteria was exceeded. The developed algorithm was tested and validated against 20 experimental baseline and 4-year follow-up Kellgren-Lawrence grades, indicating different levels of cartilage degeneration at the tibiofemoral contact region 106.

Vascular mechanobiological modelling has an added complexity of a considerable physiological pre-stress of the matrix ${ }^{107}$. This requires the definition of an attachment or deposition stretch, the stretch of new fibrils synthesised by cells when they are incorporated into 25 the matrix ${ }^{108}$. This concept was applied in the first microstructurally-based remodelling simulation of an abdominal aortic aneurysm ${ }^{37}$. This continuum model used existing constitutive equations for medial and adventitial layers ${ }^{109}$ adapted to incorporate collagen fibril recruitment. Remodelling 
was represented by coupling the rate of change of either collagen density or recruitment stretch to the difference between current fibril stretch and attachment stretch, and an axisymmetric FEA implementation predicted aneurysm expansion consistent with in vivo and other in silico models.

This concept was sophisticated, introducing triangular distribution functions to describe

5 both recruitment and attachment stretches and further to model the diffusion and reaction of factors important in collagen synthesis and degradation alongside cells to represent aneurysm development and treatment ${ }^{38}$. This computational model was able to predict the response of an animal aneurysm model to a therapeutic increase in TGF-beta.

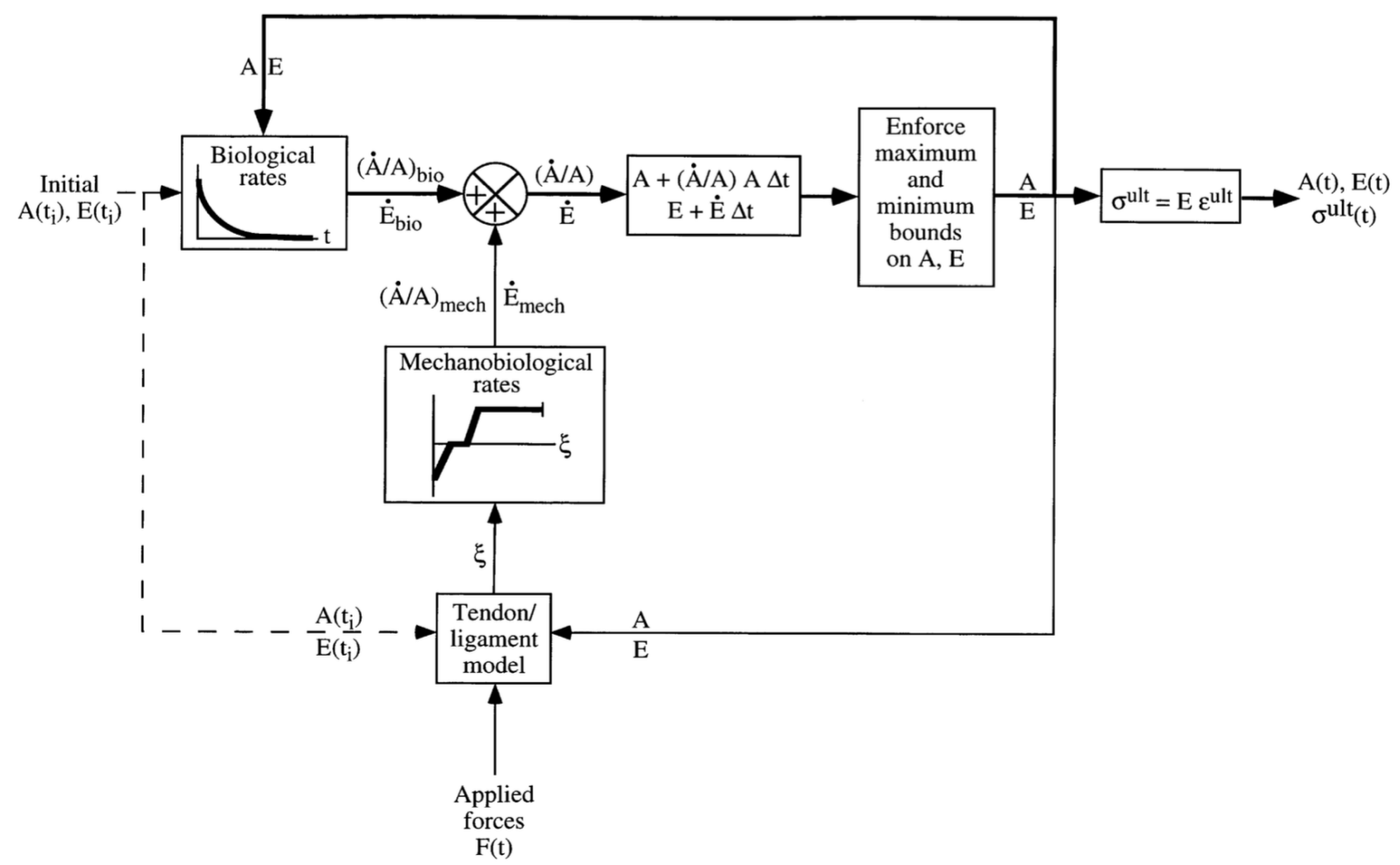

Figure 4. Analytical mechanobiological model of tendon / ligament with permission 110

A simple analytical mechanobiological model addressed the prediction of tendon crosssectional area, modulus and tensile strength under a daily strain stimulus during maturation 110 15 (Fig. 4). The rates of change were due to the sum of a decaying biological effect (aging) and the difference of the stimulus from a homeostatic value. Comparison with published data on normal, 
reduced and overloaded tendons showed reasonable agreement. Developments of the model enabled prediction of adaptation following exercise or immobilization ${ }^{111}$. An adaptive poroelastic FEA model of a tendon wrapping around a bone surface using hydrostatic pressure as a stimulus for adaptation predicted decreased permeability at the wrapping surface, suggesting tissue in the

5 region had become more cartilaginous in character 112 .

As evidenced above the wide benefits in answering clinically relevant questions that mechanobiological modelling can provide have yet to be realised in tendon. Many different modelling approaches are already available from studies of other tissues. To date, there are no modelling approaches that are optimal for tendon, and the best approach to use will depend 10 strongly upon the clinical or scientific application. Multi-scale FEA linked approaches will be valuable to represent the spatial variations expected. These will be both macroscopic in tendon healing, with multiple imaging modalities (histology, CT, ultrasound) contributing, and also microstructural, with models created with volumetric data sets imported directly from microscopic imaging modalities. These microscopic models have the greatest potential to contribute new insight

15 into tendon mechanotransduction. However, homogenised representations of these detailed microstructural mechanisms will be required in order to follow macroscopic adaptation and tissue differentiation. Therefore, approaches which represent 'averaged' fibril organisation will still have an important role to play. Finally, the potential for non-FEA approaches without detailed spatial representation is high, especially in providing fast, effective input into clinical decisions.

\section{Conclusions}

Mechanobiological modelling of tendons has the potential to revolutionise our understanding of tendon adaptation, repair and disease and to point the way to new therapies. Despite encouraging early results, the full range of computational mechanobiological modelling tools has not been applied to study tendons. Biomechanical models using the full range of data now 25 available for the microstructural environment of the tendon cell and adaptive models exploiting 
techniques developed to represent recruitment, attachment and pre-stress in vascular mechanobiology are two of the lowest hanging fruit.

This approach needs to be developed in parallel with an increased availability of high quality longitudinal data from medical imaging, biochemistry, histological and biomechanical studies either

5 directly in the clinic or using animal models. Such data is lacking for tendon adaptation, healing and degeneration and but is essential to enable validation of computational modelling frameworks to achieve clinical impact. This impact will be of increasing clinical importance to maintain musculoskeletal health in an active, aging population. Tendon can also provide a suite of easily accessible in vitro and in vivo models of a general connective tissue containing fibrillar collagen,

10 elastin and glycosaminoglycans, suitable as a first step in developing protocols and studies for more complex and less easily accessible tissues.

\section{Acknowledgements}

NMB is supported by a King's Scholarship of Malaysia. HK is supported by the European

15 Commissions's Marie Curie Intra-European Fellowship for Career Development (PIEF-GA- 2012626941).

\section{References}

1. Jarvinen M, Kannus P, Paavola M. Epidemiology of tendon problems in sport. In: Maffulli N, Renstrom P, Leadbetter WB, (eds.). Tendon Injuries: Basic Science and Clinical Medicine. London: Springer, 2005, p. 32-9.

2. Lantto I, Heikkinen J, Flinkkila T, Ohtonen P, Leppilahti J. Epidemiology of Achilles tendon 25 ruptures: increasing incidence over a 33-year period. Scand J Med Sci Sports. 2015; 25: e133-8. 
3. Thorpe CT, Clegg PD, Birch HL. A review of tendon injury: why is the equine superficial digital flexor tendon most at risk? Equine Vet J. 2010; 42: 174-80.

4. Kadler KE, Holmes DF, Trotter JA, Chapman JA. Collagen fibril formation. Biochem J. 1996; 316 ( Pt 1): 1-11.

5 5. Wang JHC. Mechanobiology of tendon. Journal of Biomechanics. 2006; 39: 1563-82.

6. Gupta HS, Seto J, Krauss S, Boesecke P, Screen HRC. In situ multi-level analysis of viscoelastic deformation mechanisms in tendon collagen. Journal of Structural Biology. 2010; 169: 183-91.

7. Screen HR, Berk DE, Kadler KE, Ramirez F, Young MF. Tendon functional extracellular matrix. J Orthop Res. 2015; 33: 793-9.

10 8. Komi PV, Fukashiro S, Jarvinen M. Biomechanical loading of Achilles tendon during normal locomotion. Clin Sports Med. 1992; 11: 521-31.

9. Ker RF, Wang XT, Pike AV. Fatigue quality of mammalian tendons. J Exp Biol. 2000; 203: 1317-27.

10. Fung DT, Wang VM, Andarawis-Puri N, et al. Early response to tendon fatigue damage 15 accumulation in a novel in vivo model. Journal of Biomechanics. 2010; 43: 274-9.

11. Lavagnino M, Wall ME, Little D, Banes AJ, Guilak F, Arnoczky SP. Tendon mechanobiology: Current knowledge and future research opportunities. J Orthop Res. 2015; 33: 813-22.

12. Thompson MS. Tendon Mechanobiology: Experimental Models Require Mathematical Underpinning. Bulletin of Mathematical Biology. 2013; 75: 1238-54.

20 13. Grant TM. Microstructural deformation of tendon. University of Oxford, 2014.

14. Handsfield GG, Slane LC, Screen HR. Nomenclature of the tendon hierarchy: An overview of inconsistent terminology and a proposed size-based naming scheme with terminology for multimuscle tendons. J Biomech. 2016.

15. Screen HRC, Bader DL, Lee DA, Shelton JC. Local Strain Measurement within Tendon. Strain. $25 \quad 2004 ; 40: 157-63$.

16. Butler DL, Goldstein SA, Guilak F. Functional Tissue Engineering: The Role of Biomechanics. Journal of Biomechanical Engineering. 2000; 122: 570-5. 
17. Thorpe CT, Riley GP, Birch HL, Clegg PD, Screen HR. Fascicles from energy-storing tendons show an age-specific response to cyclic fatigue loading. J R Soc Interface. 2014; 11: 20131058. 18. Fung Y. Elasticity of soft tissues in simple elongation. American Journal of Physiology -Legacy Content. 1967; 213: 1532-44.

5 19. Vaishnav RN, Young JT, Patel DJ. Distribution of Stresses and of Strain-Energy Density through the Wall Thickness in a Canine Aortic Segment. Circulation Research. 1973; 32: 577-83. 20. Takamizawa K, Hayashi K. Strain energy density function and uniform strain hypothesis for arterial mechanics. Journal of Biomechanics. 1987; 20: 7-17.

21. Veronda DR, Westmann RA. Mechanical characterization of skin\&\#x2014;Finite 10 deformations. Journal of Biomechanics. 1970; 3: 123-4.

22. Hirokawa S, Tsuruno R. Hyper-elastic model analysis of anterior cruciate ligament. Medical Engineering \& Physics. 1997; 19: 637-51.

23. Hirokawa S, Tsuruno R. Three-dimensional deformation and stress distribution in an analytical/computational model of the anterior cruciate ligament. Journal of Biomechanics. 2000;

15 33: 1069-77.

24. Weiss JA, Maker BN, Govindjee S. Finite element implementation of incompressible, transversely isotropic hyperelasticity. Computer Methods in Applied Mechanics and Engineering. 1996; 135: 107-28.

25. Zobitz ME, Luo Z-P, An K-N. Determination of the Compressive Material Properties of the 20 Supraspinatus Tendon. Journal of Biomechanical Engineering. 2000; 123: 47-51.

26. Hwang E, Carpenter JE, Hughes RE, Palmer ML. Shoulder labral pathomechanics with rotator cuff tears. Journal of Biomechanics. 2014; 47: 1733-8.

27. Carpenter JE, Wening JD, Mell AG, Langenderfer JE, Kuhn JE, Hughes RE. Changes in the long head of the biceps tendon in rotator cuff tear shoulders. Clinical Biomechanics. 2005; 20: 162-5.

25 28. Shim VB, Fernandez JW, Gamage PB, et al. Subject-specific finite element analysis to characterize the influence of geometry and material properties in Achilles tendon rupture. Journal of Biomechanics. 2014; 47: 3598-604. 
29. Holzapfel GA, Eberlein R, Wriggers P, Weizsäcker HW. Large strain analysis of soft biological membranes: Formulation and finite element analysis. Computer Methods in Applied Mechanics and Engineering. 1996; 132: 45-61.

30. Holzapfel GA, Gasser TC. A viscoelastic model for fiber-reinforced composites at finite

5 strains: Continuum basis, computational aspects and applications. Computer Methods in Applied Mechanics and Engineering. 2001; 190: 4379-403.

31. Gasser TC, Ogden RW, Holzapfel GA. Hyperelastic modelling of arterial layers with distributed collagen fibre orientations. Journal of The Royal Society Interface. 2006; 3: 15-35.

32. Bajuri MN, Isaksson $\mathrm{H}$, Eliasson $\mathrm{P}$, Thompson MS. A hyperelastic fibre-reinforced continuum 10 model of healing tendons with distributed collagen fibre orientations. Biomech Model Mechanobiol. 2016.

33. Driessen NJ, Cox MA, Bouten CV, Baaijens FP. Remodelling of the angular collagen fiber distribution in cardiovascular tissues. Biomech Model Mechanobiol. 2008; 7: 93-103.

34. Frisén M, Mägi M, Sonnerup L, Viidik A. Rheological analysis of soft collagenous tissue: Part I: 15 Theoretical considerations. Journal of Biomechanics. 1969; 2: 13-20.

35. Hurschler C, Loitz-Ramage B, Vanderby JR. A Structurally Based Stress-Stretch Relationship for Tendon and Ligament. Journal of Biomechanical Engineering. 1997; 119: 392-9.

36. Szczesny SE, Peloquin JM, Cortes DH, Kadlowec JA, Soslowsky LJ, Elliott DM. Biaxial Tensile Testing and Constitutive Modeling of Human Supraspinatus Tendon. Journal of Biomechanical 20 Engineering. 2012; 134: 21004-NaN.

37. Watton PN, Hill NA, Heil M. A mathematical model for the growth of the abdominal aortic aneurysm. Biomech Model Mechanobiol. 2004; 3: 98-113.

38. Aparicio P, Thompson MS, Watton PN. A novel chemo-mechano-biological model of arterial tissue growth and remodelling. J Biomech. 2016.

25 39. Shearer T. A new strain energy function for the hyperelastic modelling of ligaments and tendons based on fascicle microstructure. Journal of Biomechanics. 2015; 48: 290-7. 
40. Kastelic J, Palley I, Baer E. A structural mechanical model for tendon crimping. Journal of Biomechanics. 1980; 13: 887-93.

41. Shearer T. A new strain energy function for modelling ligaments and tendons whose fascicles have a helical arrangement of fibrils. Journal of Biomechanics. 2015; 48: 3017-25.

5 42. Guerin HA, Elliott DM. The Role of Fiber-Matrix Interactions in a Nonlinear Fiber-Reinforced Strain Energy Model of Tendon. Journal of Biomechanical Engineering. 2004; 127: 345-50.

43. Szczesny SE, Elliott DM. Interfibrillar shear stress is the loading mechanism of collagen fibrils in tendon. Acta Biomaterialia. 2014; 10: 2582-90.

44. Engelhardt C, Ingram D, Müllhaupt P, et al. Effect of partial-thickness tear on loading 10 capacities of the supraspinatus tendon: a finite element analysis. Computer Methods in Biomechanics and Biomedical Engineering. 2015: 1-8.

45. Weiss JA, Gardiner JC, Ellis BJ, Lujan TJ, Phatak NS. Three-dimensional finite element modeling of ligaments: Technical aspects. Medical Engineering \& Physics. 2005; 27: 845-61. 46. Pierrat B, Murphy JG, MacManus DB, Gilchrist MD. Finite element implementation of a new 15 model of slight compressibility for transversely isotropic materials. Computer Methods in Biomechanics and Biomedical Engineering. 2015: 1-14.

47. Fung YC. Biomechanics: Its scope, history, and some problems of continuum mechanics in physiology : Yuan-Cheng B. Fung, Appl. Mech. Rev., 21 (1) (1968) 1-20; 7 figs., many refs. Wear. 1968; $12: 137$.

20 48. Haut RC, Little RW. A constitutive equation for collagen fibers. Journal of Biomechanics. 1972; 5: 423-30.

49. Woo SLY, Gomez MA, Akeson WH. The Time and History-Dependent Viscoelastic Properties of the Canine Medial Collateral Ligament. Journal of Biomechanical Engineering. 1981; 103: 293-8. 50. Woo SL. Mechanical properties of tendons and ligaments. I. Quasi-static and nonlinear 25 viscoelastic properties. Biorheology. 1982; 19: 385-96.

51. Johnson GA, Tramaglini DM, Levine RE, Ohno K, Choi NY, Woo SL. Tensile and viscoelastic properties of human patellar tendon. J Orthop Res. 1994; 12: 796-803. 
52. Lucas SR, Bass CR, Salzar RS, et al. Viscoelastic properties of the cervical spinal ligaments under fast strain-rate deformations. Acta Biomaterialia. 2008; 4: 117-25.

53. DeFrate LE, Li G. The prediction of stress-relaxation of ligaments and tendons using the quasi-linear viscoelastic model. Biomechanics and Modeling in Mechanobiology. 2006; 6: 245-51.

5 54. Tang CY, Ng GY, Wang ZW, Tsui CP, Zhang G. Parameter optimization for the viscohyperelastic constitutive model of tendon using FEM. Biomed Mater Eng. 2011; 21: 9-24.

55. Troyer KL, Shetye SS, Puttlitz CM. Experimental Characterization and Finite Element Implementation of Soft Tissue Nonlinear Viscoelasticity. Journal of Biomechanical Engineering. 2012; 134: 114501-.

10 56. Duenwald SE, Vanderby R, Jr., Lakes RS. Stress relaxation and recovery in tendon and ligament: experiment and modeling. Biorheology. 2010; 47: 1-14.

57. Johnson GA, Livesay GA, Woo SL, Rajagopal KR. A single integral finite strain viscoelastic model of ligaments and tendons. J Biomech Eng. 1996; 118: 221-6.

58. Vena P, Gastaldi D, Contro R. A Constituent-Based Model for the Nonlinear Viscoelastic 15 Behavior of Ligaments. Journal of Biomechanical Engineering. 2005; 128: 449-57.

59. Puxkandl R, Zizak I, Paris 0, et al. Viscoelastic properties of collagen: synchrotron radiation investigations and structural model. Philosophical Transactions of the Royal Society of London B: Biological Sciences. 2002; 357: 191-7.

60. Ciarletta P, Micera S, Accoto D, Dario P. A novel microstructural approach in tendon 20 viscoelastic modelling at the fibrillar level. Journal of Biomechanics. 2006; 39: 2034-42.

61. Ciarletta P, Ben Amar M. A finite dissipative theory of temporary interfibrillar bridges in the extracellular matrix of ligaments and tendons. Journal of The Royal Society Interface. 2009; 6: 90924.

62. Sopakayang R, De Vita R. A mathematical model for creep, relaxation and strain stiffening in 25 parallel-fibered collagenous tissues. Medical Engineering \& Physics. 2011; 33: 1056-63. 
63. Khayyeri H, Gustafsson A, Heuijerjans A, et al. A fibre-reinforced poroviscoelastic model accurately describes the biomechanical behaviour of the rat Achilles tendon. PLOS ONE. 2015; 10: e0126869.

64. Khayyeri H, Longo G, Gustafsson A, Isaksson H. Comparison of structural anisotropic soft 5 tissue models for simulating Achilles tendon tensile behaviour. J Mech Behav Biomed Mater. 2016; 61: 431-43.

65. Thorpe CT, Godinho MS, Riley GP, Birch HL, Clegg PD, Screen HR. The interfascicular matrix enables fascicle sliding and recovery in tendon, and behaves more elastically in energy storing tendons. J Mech Behav Biomed Mater. 2015; 52: 85-94.

10 66. Gao F, Damsgaard M, Rasmussen J, Christensen ST. Computational method for muscle-path representation in musculoskeletal models. Biol Cybern. 2002; 87: 199-210.

67. Beskos DE, Jenkins JT. A Mechanical Model for Mammalian Tendon. Journal of Applied Mechanics. 1975; 42: 755-8.

68. Comninou M, Yannas IV. Dependence of stress-strain nonlinearity of connective tissues on 15 the geometry of collagen fibres. Journal of Biomechanics. 1976; 9: 427-33.

69. Buckley CP, Lloyd DW, Konopasek M. On the deformation of slender filaments with planar crimp: theory, numerical solution and applications to tendon collagen and textile materials. Proceedings of the Royal Society of London Series A: Mathematical, Physical and Engineering Sciences. 1980; 372: 33-64.

20 70. Stouffer DC, Butler DL, Hosny D. The Relationship Between Crimp Pattern and Mechanical Response of Human Patellar Tendon-Bone Units. Journal of Biomechanical Engineering. 1985; 107: $158-65$.

71. Lanir Y. A structural theory for the homogeneous biaxial stress-strain relationships in flat collagenous tissues. Journal of Biomechanics. 1979; 12: 423-36.

25 72. Belkoff SM, Haut RC. Microstructurally based model analysis of gamma-irradiated tendon allografts. J Orthop Res. 1992; 10: 461-4. 
73. Kwan MK, Woo SL. A structural model to describe the nonlinear stress-strain behavior for parallel-fibered collagenous tissues. J Biomech Eng. 1989; 111: 361-3.

74. Grytz R, Meschke G. Constitutive modeling of crimped collagen fibrils in soft tissues. Journal of the Mechanical Behavior of Biomedical Materials. 2009; 2: 522-33.

5 75. Goh KL, Meakin JR, Aspden RM, Hukins DWL. Influence of fibril taper on the function of collagen to reinforce extracellular matrix. Proceedings of the Royal Society of London Series B: Biological Sciences. 2005; 272: 1979-83.

76. Goh KL, Meakin JR, Aspden RM, Hukins DWL. Stress transfer in collagen fibrils reinforcing connective tissues: Effects of collagen fibril slenderness and relative stiffness. Journal of Theoretical 10 Biology. 2007; 245: 305-11.

77. Reese SP, Maas SA, Weiss JA. Micromechanical models of helical superstructures in ligament and tendon fibers predict large Poisson's ratios. Journal of Biomechanics. 2010; 43: 1394-400.

78. Ahmadzadeh H, Freedman BR, Connizzo BK, Soslowsky LJ, Shenoy VB. Micromechanical poroelastic finite element and shear-lag models of tendon predict large strain dependent Poisson's 15 ratios and fluid expulsion under tensile loading. Acta Biomaterialia. 2015; 22: 83-91.

79. Fessel G, Snedeker JG. Equivalent stiffness after glycosaminoglycan depletion in tendon--an ultra-structural finite element model and corresponding experiments. Journal of Theoretical Biology. $2011 ; 268: 77-83$.

80. Pensalfini M, Duenwald-Kuehl S, Kondratko-Mittnacht J, Lakes R, Vanderby R. Evaluation of 20 Global Load Sharing and Shear-Lag Models to Describe Mechanical Behavior in Partially Lacerated Tendons. Journal of Biomechanical Engineering. 2014; 136: 091006-.

81. Kim E, Guilak F, Haider MA. An axisymmetric boundary element model for determination of articular cartilage pericellular matrix properties in situ via inverse analysis of chondron deformation. J Biomech Eng. 2010; 132: 031011.

25 82. Lavagnino M, Arnoczky SP, Kepich E, Caballero O, Haut RC. A finite element model predicts the mechanotransduction response of tendon cells to cyclic tensile loading. Biomechanics and Modeling in Mechanobiology. 2008; 7: 405-16. 
83. Rodriguez ML, McGarry PJ, Sniadecki NJ. Review on Cell Mechanics: Experimental and Modeling Approaches. Applied Mechanics Reviews. 2013; 65: 060801-.

84. Barreto S, Clausen CH, Perrault CM, Fletcher DA, Lacroix D. A multi-structural single cell model of force-induced interactions of cytoskeletal components. Biomaterials. 2013; 34: 6119-26.

5 85. Khayyeri H, Barreto S, Lacroix D. Primary cilia mechanics affects cell mechanosensation: A computational study. J Theor Biol. 2015; 379: 38-46.

86. Ujihara Y, Nakamura M, Soga M, Koshiyama K, Miyazaki H, Wada S. Computational studies on strain transmission from a collagen gel construct to a cell and its internal cytoskeletal filaments. Computers in Biology and Medicine. 2015; 56: 20-9.

10 87. Carter DR, Wong M. The role of mechanical loading histories in the development of diarthrodial joints. Journal of Orthopaedic Research. 1988; 6: 804-16.

88. Carter DRP, Beaupre GSP, Giori NJMDP, Helms JADDSP. Mechanobiology of skeletal regeneration. $1998 ; 355:$ 41-55.

89. Lacroix D, Prendergast PJ. A mechano-regulation model for tissue differentiation during 15 fracture healing: analysis of gap size and loading. J Biomech. 2002; 35: 1163-71.

90. Lacroix D, Prendergast PJ, Li G, Marsh D. Biomechanical model to simulate tissue differentiation and bone regeneration: Application to fracture healing. Med Biol Eng Comput. 2002; 40: 14-21.

91. Isaksson H, Wilson W, van Donkelaar CC, Huiskes R, Ito K. Comparison of biophysical stimuli 20 for mechano-regulation of tissue differentiation during fracture healing. Journal of Biomechanics. 2006; 39: 1507-16.

92. Perren SM, Cordey J. The concept of interfragmentary strain. Current concepts of internal fixation of fractures1980, p. 63-77.

93. Isaksson H. Recent advances in mechanobiological modeling of bone regeneration. Mech Res 25 Commun. 2012; 42: 22-31. 
94. Khayyeri H, Isaksson H, Prendergast PJ. Corroboration of computational models for mechanoregulated stem cell differentiation. Computer Methods in Biomechanics and Biomedical Engineering. 2015; 18: 15-23.

95. Geris L, Andreykiv A, van Oosterwyck H, et al. Numerical simulation of tissue differentiation 5 around loaded titanium implants in a bone chamber. Journal of Biomechanics. 2004.

96. Isaksson H, van Donkelaar CC, Huiskes R, Ito K. A mechano-regulatory bone-healing model incorporating cell-phenotype specific activity. J Theor Biol. 2008; 252: 230-46.

97. Lacroix D, Planell JA, Prendergast PJ. Computer-aided design and finite-element modelling of biomaterial scaffolds for bone tissue engineering. Philosophical transactions Series A, Mathematical, 10 physical, and engineering sciences. 2009; 367: 1993-2009.

98. Boccaccio A, Uva AE, Fiorentino M, Lamberti L, Monno G. A Mechanobiology-based Algorithm to Optimize the Microstructure Geometry of Bone Tissue Scaffolds. International Journal of Biological Sciences. 2016; 12: 1-17.

99. Shefelbine SJ, Carter DR. Mechanobiological predictions of growth front morphology in 15 developmental hip dysplasia. J Orthop Res. 2004; 22: 346-52.

100. Giorgi M, Carriero A, Shefelbine SJ, Nowlan NC. Mechanobiological simulations of prenatal joint morphogenesis. Journal of Biomechanics. 2014; 47: 989-95.

101. Pereira AF, Javaheri B, Pitsillides AA, Shefelbine SJ. Predicting cortical bone adaptation to axial loading in the mouse tibia. J R Soc Interface. 2015; 12: 0590.

20 102. Zollner AM, Buganza Tepole A, Gosain AK, Kuhl E. Growing skin: Tissue expansion in pediatric forehead reconstruction. Biomechanics and modeling in mechanobiology. 2012; 11: 855-67. 103. Wyczalkowski MA, Varner VD, Taber LA. Computational and experimental study of the mechanics of embryonic wound healing. Journal of the Mechanical Behavior of Biomedical Materials. 2013; 28: 125-46.

25 104. Buganza Tepole A, Kuhl E. Computational modeling of chemo-bio-mechanical coupling: a systems-biology approach toward wound healing. Computer Methods in Biomechanics and Biomedical Engineering. 2016; 19: 13-30. 
105. Wilson W, Driessen NJ, van Donkelaar CC, Ito K. Prediction of collagen orientation in articular cartilage by a collagen remodeling algorithm. Osteoarthritis Cartilage. 2006; 14: 1196-202. 106. Mononen ME, Tanska P, Isaksson H, Korhonen RK. A Novel Method to Simulate the Progression of Collagen Degeneration of Cartilage in the Knee: Data from the Osteoarthritis $5 \quad$ Initiative. Sci Rep. 2016; 6: 21415.

107. Cyron CJ, Humphrey JD. Growth and remodelling of load-bearing biological soft tissues. Meccanica. 2016; in press.

108. Humphrey JD, Rajagopal KR. A constrained mixture model for growth and remodeling of soft tissues. Math Mod Meth Appl S. 2002; 12: 407-30.

10 109. Holzapfel G, Gasser T, Ogden R. A New Constitutive Framework for Arterial Wall Mechanics and a Comparative Study of Material Models. Journal of Elasticity. 2000; 61: 1-48.

110. Wren TA, Beaupre GS, Carter DR. A model for loading-dependent growth, development, and adaptation of tendons and ligaments. J Biomech. 1998; 31: 107-14.

111. Wren TA, Beaupre GS, Carter DR. Tendon and ligament adaptation to exercise, 15 immobilization, and remobilization. J Rehabil Res Dev. 2000; 37: 217-24.

112. Wren TA, Beaupre GS, Carter DR. Mechanobiology of tendon adaptation to compressive loading through fibrocartilaginous metaplasia. J Rehabil Res Dev. 2000; 37: 135-43. 